\title{
Use of robotics in spinal cord injury: a case report.
}

\author{
Lori Siedziewski \\ Moss Rehab Hospital \\ Roseann C. Schaaf \\ Thomas Jefferson University \\ Julie Mount \\ Thomas Jefferson University
}

Follow this and additional works at: https://jdc.jefferson.edu/otfp

Part of the Occupational Therapy Commons

Let us know how access to this document benefits you

\section{Recommended Citation}

Siedziewski, Lori; Schaaf, Roseann C.; and Mount, Julie, "Use of robotics in spinal cord injury: a case report." (2012). Department of Occupational Therapy Faculty Papers. Paper 34.

https://jdc.jefferson.edu/otfp/34

This Article is brought to you for free and open access by the Jefferson Digital Commons. The Jefferson Digital Commons is a service of Thomas Jefferson University's Center for Teaching and Learning (CTL). The Commons is a showcase for Jefferson books and journals, peer-reviewed scholarly publications, unique historical collections from the University archives, and teaching tools. The Jefferson Digital Commons allows researchers and interested readers anywhere in the world to learn about and keep up to date with Jefferson scholarship. This article has been accepted for inclusion in Department of Occupational Therapy Faculty Papers by an authorized administrator of the Jefferson Digital Commons. For more information, please contact: JeffersonDigitalCommons@jefferson.edu. 


\title{
Use of Robotics in Spinal Cord Injury: A Case Report
}

\author{
Lori Sledziewski, Roseann C. Schaaf, Julie Mount
}

KEY WORDS

- quadriplegia

- range of motion, articular

- robotics

- self care

- spinal cord injuries

- upper extremity
Lori Sledziewski, MS, OTR/L, is Occupational Therapist, Spinal Cord Injury Unit, Moss Rehab Hospital, 60 Township Line Road, Elkins Park, PA 19027; sledziel@einstein.edu

Roseann C. Schaaf, PhD, OTR/L, FAOTA, is Professor and Vice Chairman, Department of Occupational Therapy, Thomas Jefferson University, Philadelphia.

Julie Mount, PhD, PT, is Professor, Department of Physical Therapy, Thomas Jefferson University, Philadelphia.
OBJECTIVE. We examined the use of robotics to treat upper-extremity (UE) dysfunction in tetraplegic patients with spinal cord injury (SCI).

METHOD. A 51-yr-old man with an incomplete SCI participated in an occupational therapy program that combined traditional occupational therapy with $\mathrm{Reo} \mathrm{Go}^{\circledR}$, a comprehensive therapy platform that includes a robotic guide featuring a telescopic arm to enable high repetitions of functionally relevant UE exercises.

RESULTS. The participant demonstrated measurable improvements in active range of motion, muscle strength as measured through manual muscle testing, perceived right UE function, and self-care performance as measured by the $\mathrm{FIM}^{\mathrm{TM}}$.

CONCLUSION. The findings from this case are promising and demonstrate the Reo Go's utility in combination with traditional occupational therapy. However, more research and specific protocols that are easily reproducible with robots such as the Reo Go are needed to validate this evolving treatment area.

Sledziewski, L., Schaaf, R. C., \& Mount, J. (2012). Use of robotics in spinal cord injury: A case report. American Journal of Occupational Therapy, 66, 51-58. doi: 10.5014/ajot.2012.000943

$\mathrm{N}$ o literature supporting the use of upper-extremity (UE) robotics to treat tetraplegic patients with spinal cord injury (SCI) exists. Instead, the focus of robotic rehabilitation for the UE has mainly focused on the population with stroke. Thus, we reviewed this literature to provide a basis for the use of robotics with an individual patient with SCI. We completed two systematic reviews on the effects of robot-aided therapy on recovery of the hemiparetic UE after stroke using the PubMed, Google Scholar, CINAHL, MEDLINE, Cochrane EvidenceBased Medicine, OvidSP, and OT Search databases. Through both reviews, we found that robotic therapy improves motor control of the hemiparetic arm, yet functional improvements and gains in self-care independence were not consistent (Kwakkel, Kollen, \& Krebs, 2008; Prange, Jannink, Groothuis-Oudshoorn, Hermens, \& Ijzerman, 2006).

For example, Volpe et al. (2000) compared the use of robotic programs among 96 inpatients with stroke. The experimental group completed 25 one-hr sessions with the MIT-manus (Volpe et al., 2000), which provided a program of robotic active-assisted range-of-motion (AAROM) exercises to clients. The control group in this study performed the same tasks as the experimental group; however, the robot did not provide active assistance throughout the range of motion (ROM). Instead, the patient could assist with the unimpaired arm, or the technician in attendance could help the patient complete the movement. Despite the groups being comparable on admission, the robot-trained group demonstrated twice as much improvement as the control group in motor skills, as evidenced by Fugl-Meyer Assessment scores (Fugl-Meyer, Jääskö, Leyman, Olsson, \& Steglind, 1975).

Fasoli, Krebs, Stein, Frontera, and Hogan (2003) also examined the effects of the MIT-manus on reducing motor impairment and enhancing recovery of the hemiparetic arm in people with chronic stroke. In their initial study, Fasoli et al. found statistically significant gains from admission to discharge on 
Fugl-Meyer test Motor Status Scale scores and motor power scores. In their 2004 follow-up study, Fasoli et al. found that motor abilities of the robot-exercised limb were maintained $4 \mathrm{mo}$ after discharge. Another study (Krebs et al., 2008) provided evidence that short-term, goal-directed robotic therapy using the MIT-manus can result in improved motor abilities in people with chronic stroke.

Another interesting trend noted in the literature was that of studies that examined the effects of robotic therapy and conventional therapy for patients with stroke. Lum Burgar, Shor, Majmundar, and Van der Loos (2002) completed a randomized controlled trial with 27 participants with hemiparesis, all at least 6 mo poststroke. Lum et al. compared the control group, which received conventional neurodevelopmental treatment, with the experimental group, which received 24 one-hr sessions during which they engaged in exercises produced by the Mirror Image Movement Enabler robot. Results of this study showed that the robot group had larger gains in strength and reach extent after 2 mo. Moreover, at the 6-mo follow-up, the robot group maintained larger improvements in FIM ${ }^{\mathrm{TM}}$ (Hamilton, Granger, Sherwin, Zielezny, \& Tashman, 1987) scores than the control group (Lum et al., 2002). In another study, Nef, Quinter, Muller, and Riener (2009) found improvements in motor recovery as measured by increased active range of motion (AROM) in the hemiparetic UE in three single cases after using the ARMin I robot.

Projecting into the future, Reinkensmeyer, Pang, Nessler, and Painter (2002) discussed potential trends in robotic therapy use in the population with stroke. They hypothesized that the future of robotic rehabilitation may include use of Java Therapy (Reinkensmeyer, Painter, Yang, Abbey, \& Kaino, 2000), which involves robotic therapy at home through the use of a library of evaluation and therapy activities that can be accessed through a therapy program's Web page. This advancing technology allows people to engage in additional functionally meaningful tasks at home in addition to traditional therapy in the clinic, thus increasing engagement in a wide variety of therapeutic activities.

\section{Reo Go ${ }^{\circledR}$ System and Stroke}

Only a few studies have focused exclusively on the Reo Go system (Motorika Medical, Caesarea, Israel). The Reo Go is a comprehensive therapy platform that includes a robotic guide featuring a telescopic arm to deliver many repetitions of functionally relevant UE exercises. Padova, Werner, Mahoney, and Esquenazi (2007) completed a pilot trial using the Reo Go to treat 10 people with hemiparesis resulting from stroke. Results indicated a reduction in perceived exertion, reduction in shoulder pain, and reduction in spasticity, as measured on the Ashworth scale (Bohannon \& Smith, 1987). Moreover, all 10 participants demonstrated improvement on the FuglMeyer, with scores increasing by between 2 and 11 points. Treger, Faran, and Ring (2008) also found that arm impairment and functionality as measured by the Fugl-Meyer assessment and the Manual Function Test (Moriyama, 1987) increased significantly among people with chronic stroke after 15 Reo Go sessions. Neither study reported the specific exercise protocols that were used to treat people using the Reo Go.

\section{Robotics and Spinal Cord Injury}

Despite a lack of evidence for the use of UE robotics in the population with SCI, several studies have been completed on the use of robotics to elicit lower-extremity (LE) motor return in patients with incomplete SCI. These studies are based on the Central Pattern Generator Theory (Grillner, 1979, 1985; Pearson \& Rossignol, 1991). According to the theory, repetitive movements can stimulate motor recovery even in the absence of complete central nervous system innervation. Central pattern generators consist of relatively small and autonomous neural networks that, when stimulated during specific repetitive movements, can produce rhythmic movement patterns, even in the absence of motor and sensory feedback from the arms or legs (Barrière, Leblond, Provencher, \& Rossignol, 2008).

Body-weight-supported treadmill training (BWSTT; Lovely, Gregor, Roy, \& Edgerton, 1986) was introduced in the 1980s to treat incomplete SCI. Based on Central Pattern Generator Theory, BWSTT creates repetitive gait patterns over a treadmill with the hope that motor recovery can be stimulated in patients with incomplete SCI (Barrière et al., 2008). One example of a BWSTT system is the Lokomat (Hocoma, Inc., Norwell, MA). The Lokomat is a combined treadmill and gait-driven orthosis used to treat locomotor dysfunction in patients with SCI. General themes found in BWSTT research include documenting increases in LE muscular activity as recorded using electromyography during gait (Gorassini, Norton, Nevett-Duchcherer, Roy, \& Yang, 2009), improvement in gait pattern (Dobkin et al., 2003; Field-Fote \& Tepavac, 2002; Hornby, Zemon, \& Campbell, 2005), and success with transitioning to walking on other surfaces after completing BWSTT (Hornby et al., 2005).

Although the literature to support the use of robotics to treat UE dysfunction resulting from incomplete SCI is 
lacking, the evidence of its use after stroke is intriguing. In addition, the Central Pattern Generator Theory for LE movement in SCI provided an adequate theoretical rationale showing that BWSTT assists with motor return for LE function in patients with incomplete SCI. Thus, the idea of using the Reo Go, which uses common UE movements (e.g., hand to mouth) in repetitive exercise patterns, to elicit possible central pattern generators in the UE seemed feasible.

\section{Case Report Research Question}

Will an occupational therapy program that includes selfcare training and use of the Reo Go increase AROM, strength, perceived right UE function, and self-care performance in a 51-yr-old man with incomplete SCI?

\section{Case Description}

M.R. was a 51-yr-old right-handed White man who was a passenger in a motor vehicle crash. He underwent a C4-C5 anterior cervical discectomy and fusion in addition to a C3-C6 posterior laminectomy with fusion. M.R. also underwent a vertebral artery dissection. Initially, M.R. had a speech therapy evaluation and was determined to have a mild traumatic brain injury with deficits in shortterm memory, although symptoms resolved after several sessions, and he was discharged from speech services. M.R. experienced ongoing pain in his right shoulder, generally ranging from 6 to 7 out of a possible score of 10 on a numeric rating scale. Although he frequently complained of pain, it did not affect performance to a point that would discontinue his participation in a robotic exercise program. Pain was an issue discussed weekly in rounds and medically managed by the attending physician. Past medical history included alcohol abuse, barbiturate abuse, hepatitis $\mathrm{C}$, meningitis, hyponatremia, and an Achilles tendon repair.

Using the American Spinal Injury Association Impairment Scale (AIS; Maynard et al., 1977), M.R. was defined as having a C4 AIS D injury. An AIS D classification indicates that M.R. had preserved sensory and motor function below the neurological level and that at least half of the key muscles below the neurological level had a muscle grade of $\geq 3$ (Maynard et al., 1997). On admission, M.R.'s AIS motor score for upper limbs was 39 of 50; his motor score for lower limbs was 41 of 50 . His AIS light touch score was 90 of 112, and his pinprick score was 86 of 112 . He presented with right UE and left LE weakness and decreased AROM. However, his left UE presented with full AROM for all motions, and manual muscle testing (MMT) scores were 4-5 of 5 for all shoulder, elbow, and wrist movements. M.R. did not have subluxation or any instability in the right shoulder that would have made his participation in a robotic exercise program unsafe.

Before admission, M.R. was independent in all areas of self-care and instrumental activities of daily living (IADLs), including driving. He lived with his wife in a two-story duplex. After discharge, M.R. planned to return home to the duplex's first-floor apartment to limit time spent on stairs for safety. Before his accident, M.R. had worked as a roofer in the spring and summer months, but after discharge he planned to assist his wife with her online travel agency business. His leisure interests included watching sports, going to bars, and socializing. M.R.'s goals included walking around normally and not needing help to do things for himself.

\section{Assessments}

Goniometry measurements, MMT, and sensory testing including light touch and sharp-dull testing were completed at admission, midpoint, and discharge to evaluate AROM and passive ROM (PROM), strength, and sensory awareness. AROM was measured against gravity in a standing position at admission, midpoint, and discharge. M.R. was required to hold the position while the therapist measured with a goniometer. No hands-on assistance was provided for maintaining joint positioning during measuring. MMT was completed for general motions and not for individual muscles. Light touch and sharpdull were assessed in all dermatomes of the UE (C3-T1). Intact sensation was defined as identification of at least three correct sensations with no errors. Impaired sensation was defined as interpretation of at least one of three sensations incorrectly.

In addition, the Capabilities of Upper Extremity instrument (CUE; Marino, Shea, \& Stineman, 1998) was completed retrospectively to assess perceived changes in right UE function. The CUE is 32-item questionnaire developed to assess difficulty in performing certain actions with one or both arms and hands in people with tetraplegia. Responses are given on a 7-point scale representing self-perceived difficulty in performing the action $(1=$ unable to perform and $7=$ can perform without difficulty; Marino et al., 1998). The instrument displays good levels of homogeneity, test-retest reliability, and construct validity (Marino et al., 1998).

To assess changes in areas of self-care, including eating, grooming, bathing, dressing (UE and LE), toileting, and functional transfers (bed, toilet, tub), we used the FIM. FIM scores were calculated daily after selfcare treatment sessions were completed. 


\section{Outcomes}

Primary outcomes measured were changes in right UE AROM, changes in independence in self-care as measured by the FIM, and perceived changes in right UE function as measured by the CUE. Secondary outcomes included changes in strength and sensation. After completing the initial evaluation, functional goals were created but modified throughout M.R.'s hospital stay to reflect changes in independence and UE function.

\section{Intervention}

M.R. spent 20 consecutive days in inpatient rehabilitation; he received no occupational therapy treatment on the weekends for insurance reasons. His initial injury and surgical intervention occurred 26 days before his first day of inpatient rehabilitation. On his first day of rehabilitation, a brief interview, evaluation of self-care performance, and UE assessments were completed. M.R. initiated self-care training and Reo Go exercises on the second day of his admission. M.R.'s rehabilitation plan included $2.0 \mathrm{hr}$ of occupational therapy, $1.0 \mathrm{hr}$ of physical therapy, and $0.5 \mathrm{hr}$ of recreational therapy daily. One hour of M.R.'s occupational therapy was spent on daily self-care, which included education and cueing during breakfast, showering, dressing, and grooming. This session progressed from education and cueing in compensatory strategies for self-care completion, including use of a hemistrategy for UE dressing and onehanded techniques for opening containers, to active use of bilateral UEs during functional tasks as motor function in the right UE improved. For example, he was instructed to eat and drink using the right UE during meals and to open containers using bilateral UEs rather than the onehanded techniques.

M.R. spent the second hour of occupational therapy engaging in a Reo Go exercise program. The Reo Go is a comprehensive therapy platform that includes a robotic guide featuring a telescopic arm to enable high repetitions of functionally relevant arm exercises. The Reo Go's computer software customizes exercise patterns and measures performance. Initially developed in Israel, the Reo Go was introduced to the U.S. market in 2006. The Reo Go features five levels of resistance. The guided level involves no active participant involvement; only PROM is completed. At the initiated level, the participant must initiate movement; however, the remainder of movement is passive. The step-initiated level is similar to the initiated level, except that the participant must initiate movement in multiple areas throughout the segment. The fourth level, follow-assist, occurs when the arm moves toward the target and, as the patient applies force in the correct direction, the speed increases. Finally, the free level requires the participant to actively move the arm without any assistance from the robot. Movement combinations that can be elicited by the Reo Go include (1) forward reach, which elicits shoulder abduction and flexion; (2) forward thrust, which elicits shoulder flexion, elbow flexion, and extension; (3) horizontal reach, which elicits shoulder abduction and flexion and elbow flexion and extension; and (4) hand to mouth, which elicits shoulder flexion and elbow flexion and extension.

\section{M.R.'s Reo Go Program}

As specified in the Reo Go manual, M.R. engaged in a preintervention assessment to determine the initial resistance level or baseline. M.R.'s baseline level was 3 sets of 10 repetitions for each exercise at the guided level, yet M.R. was unable to complete the repetitions during the treatment sessions because of pain and fatigue levels. As noted, M.R.'s daily baseline pain was usually 6-7 of 10 in the right UE, a level that often limited his use of the Reo Go. For example, during the first session, M.R. was unable to complete his baseline 3 sets of 10 repetitions of the four guided exercises; therefore, repetitions were reduced to 3 sets of 5 for forward reach, horizontal reach, and hand to mouth. One set of 10 forward thrusts was completed.

After the Reo Go procedures, we assessed the ability to increase resistance level each day by asking the patient to complete 1 set of 10 exercises at the next level. Once he was able to do this, all exercises were then performed at the next highest level. By his 7 th occupational therapy session, M.R. was able to complete 1 set of 10 at the initiated level for all four movements; therefore, all exercises were increased to this level. Repetitions remained the same to maintain consistency. M.R. completed a total of 6 sessions at the guided level and 12 sessions at the initiated level.

\section{Results}

M.R. demonstrated improvements on all outcome measures. Specifically, he demonstrated (1) increases in AROM (Figure 1), (2) increased independence in self-care (Figure 2 ), (3) increases in strength (Figure 3), and (4) increases in perceived right UE function (Figure 4). As shown in Figure 1, M.R. demonstrated increased AROM for elbow flexion, elbow extension, shoulder internal rotation, and shoulder external rotation, although we noted no changes in AROM for right shoulder flexion and abduction. As shown in Figure 2, changes in FIM scores indicate that M.R. made improvements in self-care in every area. More specifically, M.R. improved in UE dressing and eating. 


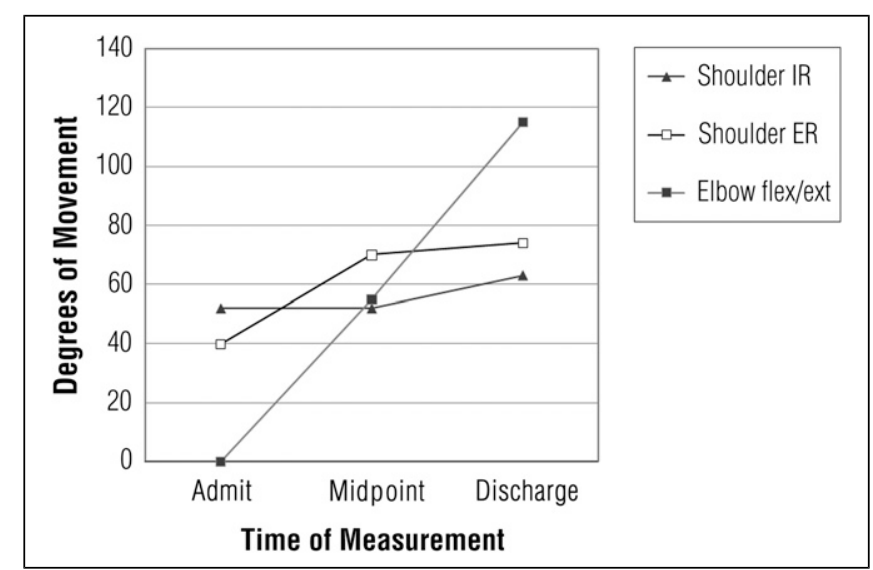

Figure 1. Right upper-extremity increases in active range of motion. The following movements did not demonstrate any change: Shoulder flexion, shoulder abduction, pronation, supination, wrist flexion, and wrist extension.

Note. $\mathrm{ER}=$ external rotation; flex/ext $=$ flexion/extension; $\mathrm{IR}=$ internal rotation.

Increases in strength were noted for shoulder internal rotation, shoulder external rotation, and elbow flexion and extension (see Figure 3). In addition, M.R. perceived that his right UE function improved (Figure 4), especially for movements involving the elbow, wrist, and digits. Although we noted no AROM in shoulder flexion and abduction, M.R. did demonstrate a muscle grade of 1 for shoulder flexion. No consistent changes in sensory function were noted.

\section{Discussion}

We noted several interesting findings in this case report. First, the finding that M.R. did demonstrate improvement in UE AROM suggests that the combination of traditional occupational therapy and use of the Reo Go did affect AROM. This finding implies that this combination of therapy was helpful for this client and supports further study of the Reo Go with the population with SCI.
Second, increases in AROM were accompanied by improvements in FIM scores, suggesting that for this client, improved AROM may have had an impact on functional skills. Although determining what aspects of the combined therapy had the greatest impact is impossible, one important finding is that this type of combined therapy may be useful in improving functional skills. Future studies should evaluate the effects of traditional occupational therapy separately and in combination with the Reo Go to determine whether the Reo Go adds significantly to gains in functional skills.

Another interesting trend noted in this case report is M.R.'s perceived areas of improvement in right UE function. As seen in Figure 4, M.R. perceived increased function in all areas between admission and discharge. The largest areas of perceived improvement were noted for pushing and pulling movements and hand and finger movements. These results correlate with improvements in AROM, with the greatest area of improvement in AROM being elbow flexion and extension, suggesting that M.R.'s perceptions were consistent with the objective data. Moreover, the motions for which M.R. perceived the most improvement were also important for self-care completion (i.e., eating, UE dressing, LE dressing, and toileting). Therefore, improvements in FIM scores in these stated areas could also be correlated with M.R.'s perceived areas of most improved function.

\section{Limitations}

Because this is a case report, the findings cannot be generalized to others; rather, they provide preliminary data that support the need for further studies in this area. Subjectivity may have altered the results because we were invested in the client's success. Another limitation of subjectivity is the decreased reliability and validity of

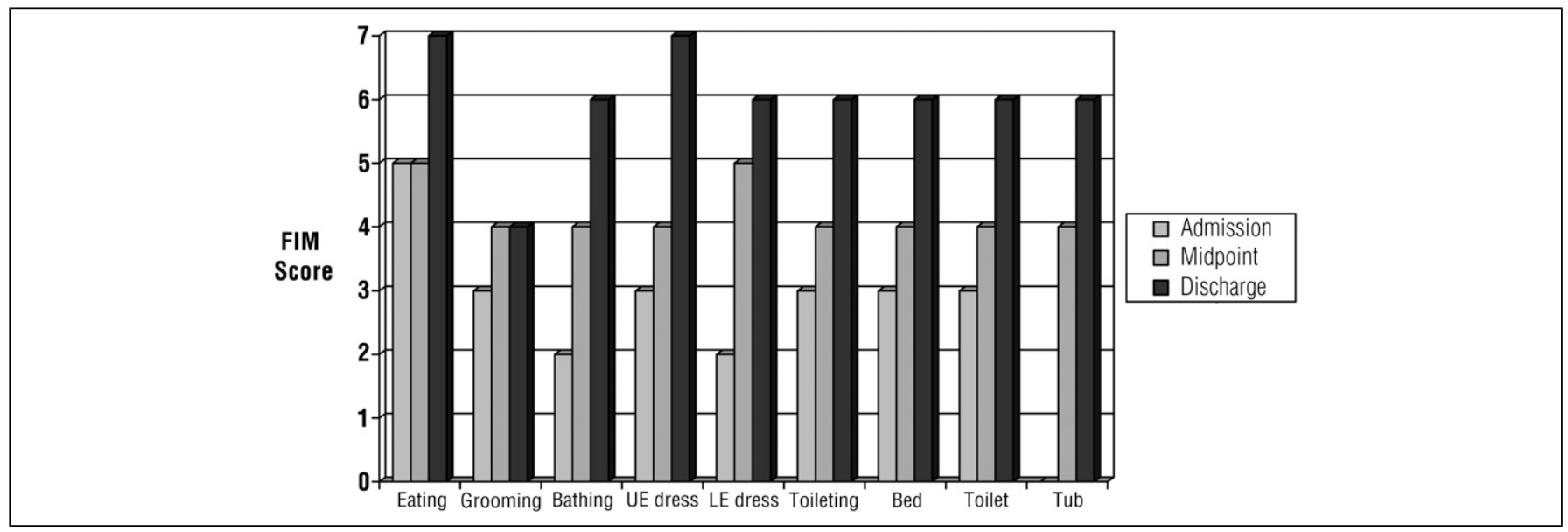

Figure 2. Self-care FIM scores at admission, midpoint, and discharge.

Note. $\mathrm{D} / \mathrm{C}=$ discharge; $\mathrm{LE}=$ lower extremity; UE = upper extremity. 


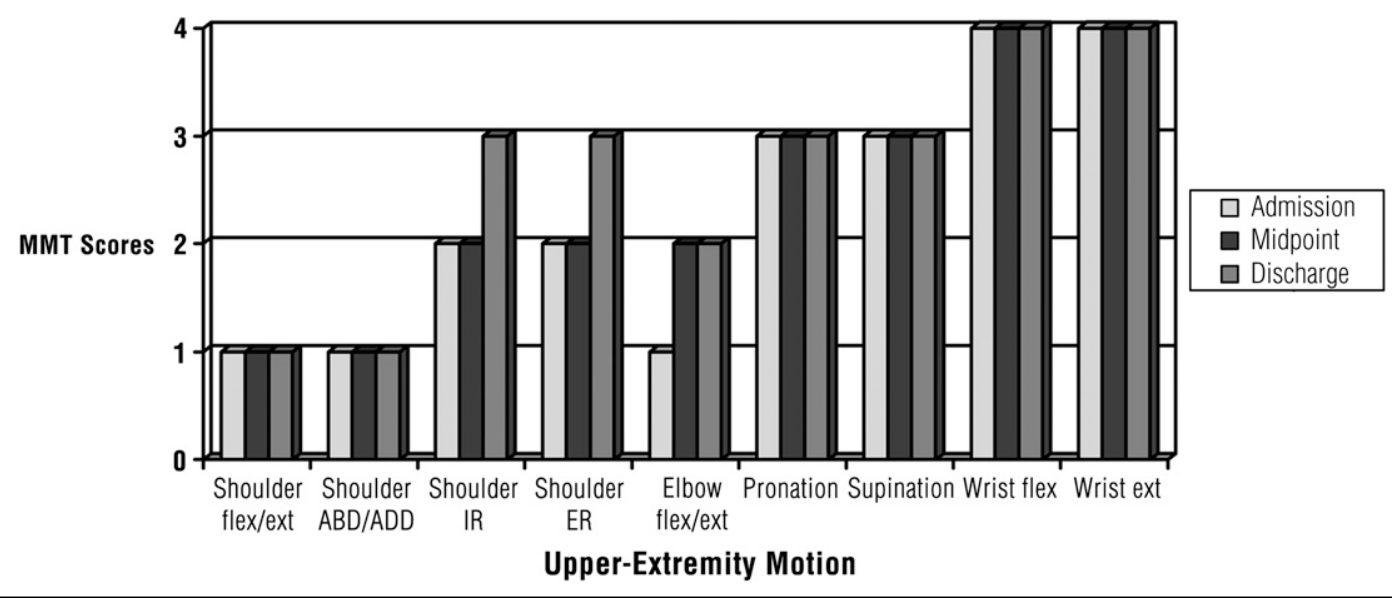

Figure 3. Changes in right upper-extremity strength.

Note. $\mathrm{ABD} / \mathrm{ADD}=$ abduction/adduction; $\mathrm{ER}=$ external rotation; flex/ext $=$ flexion/extension; $\mathrm{IR}=$ internal rotation; MMT = manual muscle testing.

goniometry measurements. In general, the literature has shown $\pm 5^{\circ}$ interrater reliability for measuring degrees of motion using a goniometer (Marx, Bombardier, \& Wright, 1999; Van Trijffel, Van de Pol, Oostendorp, \& Lucas, 2010). In this case report, the same evaluator (L. Sledziewski) completed all the admission, midpoint, and discharge goniometry measurements. In addition, we used the universal full-circle goniometer, which one study has found to be the preferred instrument to improve validity and reliability for measuring ROM (Gajdosik \& Bohannon, 1987). Despite the variability of goniometry measurements in general, we applied the necessary means to ensure the most valid and reliable ROM measurements possible.

Another limitation is that the CUE was completed retrospectively and should therefore be interpreted cautiously. More accurate perceptions could possibly have been made if the CUE had been administered at the time of admission in addition to discharge. Determining whether natural recovery or recovery from use of the Reo Go was responsible for increased AROM in the right arm was difficult. Finally, given that traditional occupational therapy was provided in combination with the Reo Go, determining whether the effects found were the result of the combined therapy or one aspect of the intervention (traditional occupational therapy vs. the Reo Go) is impossible. Further research is required to determine the active ingredients for producing the noted changes.

In retrospect, several aspects of this research, if changed, could have improved the delivery of treatment, outcomes, or both. First, trunk stabilization was not accounted for during completion of the Reo Go exercise program. If M.R.'s trunk was stabilized in the chair and trunk flexion and extension were prevented, movement could possibly have been more focused on the shoulder.
Another limitation of the Reo Go is that several movements are limited by the device itself. For example, the robot limited shoulder flexion to $80^{\circ}$ and shoulder abduction to $90^{\circ}$. However, full elbow flexion and extension ranges were available. This limitation may have affected M.R.'s AROM recovery.

Another salient aspect of this case that could have been changed was structuring the repetitions and resistance levels of the Reo Go exercises more uniformly. Currently, no research exists on Reo Go exercise protocols. In this case report, decisions on the repetitions and resistance levels were based on our clinical judgment. A more systematic way of developing the exercise protocol would have increased the reproducibility of this case report on a larger scale.

\section{Implications for Occupational Therapy Practice}

The results of this study have the following implications for occupational therapy practice:

- Upper-extremity robotics are a beneficial tool that can be used in combination with traditional occupational therapy for treating UE dysfunction in the SCI population.

- Incorporating new technology in occupational therapy treatment is important to the evolution and progression of our field.

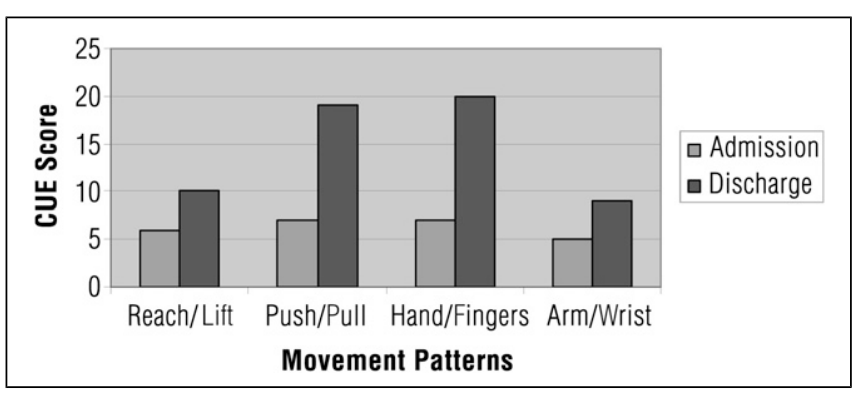

Figure 4. Perceived increase in right upper-extremity function. Note. CUE $=$ Capabilities of Upper Extremity instrument. 


\section{Conclusion}

The participant in this case report demonstrated improvements in AROM, increased independence in selfcare tasks, improved strength, and increased perceived capabilities of the right UE. However, more research is needed to support the use of robotics to treat patients with SCI. Protocols that are easily reproducible in robots such as the Reo Go are needed to validate the use of this evolving treatment area in the population with SCI, and their use in occupational therapy requires explication. Most important, future research should be designed to more clearly identify the active ingredients of recovery.

\section{Acknowledgments}

This study was completed in partial fulfillment of an advanced-practice Certificate in Neurorehabilitation at Thomas Jefferson University, Philadelphia. We thank the client for his participation and the staff and administration at Moss Rehab Hospital for their support of this project.

\section{References}

Barrière, G., Leblond, H., Provencher, J., \& Rossignol, S. (2008). Prominent role of the spinal central pattern generator in the recovery of locomotion after partial spinal cord injuries. Journal of Neuroscience, 28, 3976-3987. doi: 10.1523/JNEUROSCI.5692-07.2008

Bohannon, R. W., \& Smith, M. B. (1987). Inter-rater reliability of a modified Ashworth scale of muscle spasticity. Physical Therapy, 67, 206-207.

Dobkin, B., Apple, D., Barbeau, H., Basso, M., Behrman, A., Deforge, D., et al. (2003). Methods for a randomized trial of weight-supported treadmill training versus conventional training for walking during inpatient rehabilitation after incomplete traumatic spinal cord injury. Neurorehabilitation and Neural Repair, 17, 153-167.

Fasoli, S. E., Krebs, H. I., Stein, J., Frontera, W. R., \& Hogan, N. (2003). Effects of robotic therapy on motor impairment and recovery in chronic stroke. Archives of Physical Medicine and Rehabilitation, 84, 477-482. doi: 10.1053/ apmr.2003.50110

Fasoli, S., Krebs, H., Stein, J., Frontera, W., Hughes, R., \& Hogan, N. (2004). Robotic therapy for chronic motor impairments after stroke: Follow up results. Archives of Physical Medicine and Rehabilitation, 85, 1106-1111.

Field-Fote, E., \& Tepavac, D. (2002). Improved intralimb coordination in people with incomplete spinal cord injury following training with body weight supported and electrical stimulation. Physical Therapy, 82, 707-715.

Fugl-Meyer, A. R., Jääskö, L., Leyman, I., Olsson, S., \& Steglind, S. (1975). The poststroke hemiplegic patient: A method for evaluation of physical performance. Scandinavian Journal of Rehabilitation Medicine, 7, 13-31.

Gajdosik, R. L., \& Bohannon, R. W. (1987). Clinical measurement of range of motion: Review of goniometry em- phasizing reliability and validity. Physical Therapy, 67, 1867-1872.

Gorassini, M., Norton, J., Nevett-Duchcherer, J., Roy, F., \& Yang, J. (2009). Changes in locomotor muscle activity after treadmill training in subjects with incomplete spinal cord injury. Journal of Neurophysiology, 101, 969-979.

Grillner, S. (1979). Interaction between central and peripheral mechanisms in the control of locomotion. Progress in Brain Research, 50, 227-235.

Grillner, S. (1985). Neurobiologial bases of rhythmic motor acts in vertebrates. Science, 228, 143-149.

Hamilton, B. B., Granger, C. V., Sherwin, F. S., Zielezny, M., \& Tashman, J. S. (1987). A uniform national data system for medical rehabilitation. In M. Fuhrer (Ed.), Rehabilitation outcomes: Analysis and measurement (pp. 137-147). Baltimore: Paul H. Brookes.

Hornby, T., Zemon, D., \& Campbell, D. (2005). Roboticassisted, body-weight supported treadmill training in individuals following motor incomplete spinal cord injury. Physical Therapy, 85, 53-66.

Krebs, H. I., Mernoff, S., Fasoli, S. E., Hughes, R., Stein, J., \& Hogan, N. (2008). A comparison of functional and impairment-based robotic training in severe to moderate chronic stroke: A pilot study. NeuroRehabilitation, 23, 81-87.

Kwakkel, G., Kollen, B. J., \& Krebs, H. I. (2008). Effects of robot-assisted therapy on upper limb recovery after stroke: A systematic review. Neurorehabilitation and Neural Repair, 22, 111-121. doi: 10.1177/1545968307305457

Lovely, R. G., Gregor, R. J., Roy, R. R., \& Edgerton, V. F. (1986). Effects of training on the recovery of full weight bearing stepping in the adult spinal cat. Experimental Neurology, 92, 421-435.

Lum, P. S., Burgar, C. G., Shor, P. C., Majmundar, M., \& Van der Loos, M. (2002). Robot-assisted movement training compared with conventional therapy techniques for the rehabilitation of upper-limb motor function after stroke. Archives of Physical Medicine and Rehabilitation, 83, 952-959. doi: 10.1053/apmr.2001.33101

Marino, R., Shea, J., \& Stineman, M. (1998). The capabilities of upper extremity instrument: Reliability and validity of a measure of functional limitation in tetraplegia. Archives of Physical Medicine and Rehabilitation, 79, 1512-1521.

Marx, R., Bombardier, C., \& Wright, J. (1999). What do we know about the reliability and validity of physical examination tests used to examine the upper extremity? Journal of Hand Surgery, 24A, 185-193.

Maynard, F. M., Jr., Bracken, M. B., Creasey, G., Ditunno J. F., Jr., Donovan, W. H., Ducker, T. B., et al.; American Spinal Injury Association. (1997). International standards for neurological and functional classification of spinal cord injury. Spinal Cord, 35, 266-274. doi: 10.1038/sj.sc. 3100432

Moriyama, S. (1987). Occupational therapy in stroke rehabilitation - with reference to early stage program. In Proceedings of the Joint Japanese-China Stroke Conference (pp. 114-124). Ikarigaseki, Japan: Reimeikyo Rehabilitation Hospital.

Nef, T., Quinter, G., Muller, R., \& Riener, R. (2009). Effects of arm training with the robotic device ARMin $\mathrm{I}$ in 
chronic stroke: Three single cases. Neurodegenerative Disease, 6, 240-251.

Padova, J., Werner, L., Mahoney, R., \& Esquenazi, A. (2007). Pilot trial of a robot-assisted upper limb therapy system. Unpublished manuscript, Moss Rehab, Philadelphia.

Pearson, K. G., \& Rossignol, S. (1991). Fictive motor patterns in chronic spinal cats. Journal of Neurophysiology, 66, 1874-1887.

Prange, G. B., Jannink, M. J., Groothuis-Oudshoorn, C. G., Hermens, H. J., \& Ijzerman, M. J. (2006). Systematic review of the effect of robot-aided therapy on recovery of the hemiparetic arm after stroke. Journal of Rehabilitation Research and Development, 43, 171-184. doi: 10. 1682/JRRD.2005.04.0076

Reinkensmeyer, D. J., Painter, C., Yang, S., Abbey, E., \& Kaino, B. (2000, March). An Internet-based, force-feedback rehabilitation system for arm movement after brain injury. Paper presented at the California State UniversityNorthridge 15th Annual International Conference: Technology and Persons With Disabilities, Los Angeles.
Retrieved February 21, 2009, from www.csun.edu/cod/ conf2000/proceedings/0080Reinkensmeyer.html

Reinkensmeyer, D., Pang, C., Nessler, J., \& Painter, C. (2002). Java therapy: Web-based telerehabilitation for the upper extremity after stroke. IEEE Transactions on Neural Systems and Rehabilitation Engineering, 10, 102-108.

Treger, I., Faran, S., \& Ring, H. (2008). Robot-assisted therapy for neuromuscular training of sub-acute stroke patients: A feasibility study. European Journal of Physical and Rehabilitation Medicine, 44, 431-435.

Van Trijffel, E., Van de Pol, R., Oostendorp, R., \& Lucas, C. (2010). Inter-rater reliability for measurement of passive physiological movements in lower extremity joints is generally low: A systematic review. Journal of Physiotherapy, 56, 223-235.

Volpe, B. T., Krebs, H. I., Hogan, N., Edelstein, L., Diels, C., \& Aisen, M. (2000). A novel approach to stroke rehabilitation: Robot-aided sensorimotor stimulation. Neurology, 54, 1938-1944. 